\title{
IMPACT OF CSI LATENCY ON HF MULTI-CARRIER COMMUNICATIONS FOR DELAY-SENSITIVE APPLICATIONS
}

\author{
J. López-Pérez*, I.A. Pérez-Álvarez*, S. Zazo-Bello ${ }^{\dagger}$, L.B. Melián-Gutiérrez*, A. Amaro-García* \\ *Universidad de Las Palmas de Gran Canaria (ULPGC), CeTIC, Dpto. de Señales y Comunicaciones, Campus de Tafira, \\ 35017 - Las Palmas, Spain, Tel.: +34-928457372, e-mail: jlopez@cetic.eu \\ ${ }^{\dagger}$ Universidad Politécnica de Madrid (UPM), Dpto. SSR, ETSI de Telecomunicación-UPM, Ciudad Universitaria, 28040 - \\ Madrid, Spain, Tel.: +34-915495700 ext.4042, e-mail: santiago@gaps.ssr.upm.es
}

Keywords: Ionospheric channel, OFDM, singular value decomposition, bit-loading.

\begin{abstract}
Multi-carrier modulations are widely used in HF communications, and particularly OFDM. Delay-sensitive applications can make use of spreading schemes such as OFDM-CDM in order to cope with deep nulls in the channel. If CSI is known at transmitter, it is possible to improve performance using a bit-loading algorithm for OFDM and channel matrix SVD decomposition for OFDM-CDM. If a source of non-ideality in CSI due to latency is taken into consideration, it is shown how performance decreases. A receiver-side technique is applied to OFDM-CDM communications to compensate for delay-incurred matrices mismatch.
\end{abstract}

\section{Introduction}

Single-carrier modulations have been the traditional mean of carrying out communications in the HF band of the ionospheric channel. Because of selective fading, parts of the band suffer different magnitude and phase modifications than others. Amongst the effects causing selective fading is multipath. The bandwidth where fading can be considered as flat is known as channel frequency coherence.

Deep nulls are also caused by the time varying characteristics of the ionospheric channel, due mainly to the changing nature of the channel. Those nulls, in turn, can cause burst errors in the communication. Duration of such errors is given by the channel time coherence, or its inverse doppler spread.

In order to cope with selective fading, nowadays HF communications make extensive use of multi-carrier modulations [8]. These kinds of modulations make use of more than one carrier to transmit data, each sub-carrier being modulated to a lesser rate than would be necessary to transmit their joint information by means of a single-carrier modulation. Thus, bandwidth channel can be considered as flat for each sub-carrier.
The most extended amongst the different multi-carrier techniques is Orthogonal Frequency Division Multiplex (OFDM). Intercarrier Interference (ICI) can be avoided thanks to orthogonality amongst sub-carriers, while the use of cyclic pre- and post-fixes eliminates Intersymbol Interference (ISI). Thus, combination of sub-carriers bandwidth below frequency coherence bandwidth and usage of cyclic prefixes, guarantees availability of a strictly flat equivalent channel. Furthermore, OFDM can be easily generated in modern hardware or software by means of Discrete Fourier Transform (DFT).

However, deep nulls can attenuate one or more sub-carriers so much that information can no longer be recovered. Classical techniques to mitigate this effect are channel coding and interleaving, widely known as Coded OFDM (COFDM). These techniques are generally not well suited to delaysensitive applications, though. Channel coding adds redundancy by turning the bits to be transmitted into a higher number of symbols, whilst interleaving assigns these symbols to sub-carriers apart enough, both in time and frequency. The longer interleaver depth, the longer error burst duration the system will be able to cope with. Yet, as interleaver depth grows, so does communication delay.

Another way to avoid deep nulls effects whilst keeping delay at minimum is to apply spreading schemes over OFDM, resulting in OFDM Code Division Multiplex (OFDM-CDM) [1]. This technique makes use of orthogonal codes, such as Walsh-Hadamard codes, to distribute the transmitted symbols over all or a subset of the available OFDM sub-carriers. As each sub-carrier transports only part of a symbol, even if a deep null renders the information of a sub-carrier impossible to retrieve, the symbols may possible be recovered by the joint contribution of the surviving sub-carriers. Multiple Access Interference (MAI) is a spreading disadvantage, usually requiring Multiple User Detectors (MUD) at receiver [6].

In the HF channel, performance of COFDM has already been studied, and it has been shown to clearly outperform both OFDM and OFDM-CDM [9]. However, as previously stated, channel coding and interleaving is generally unsuitable for 
delay-sensitive applications. Thus, COFDM is not further pursued in this paper.

If Channel State Information (CSI) is known at transmitter, it is possible to precode the signal to be transmitted in order to improve performance without increasing delay.

The study carried out in this paper is part of the researchers' ongoing efforts in digital communications in the HF band. Within this research line, the prototype of a digital modem intended for both interactive voice and data communication in the HF band has been developed. Thus, the aim of this paper is to assess the possibility of applying CSI knowledge to precode the transmitted signal in order to improve performance measured as BER achieved. Moreover, a source non-ideality in channel acquisition by transmitter will be investigated. Finally, conclusions regarding which of the techniques under study are best suited for the environment considered are drawn.

\section{Precoding based on Channel State Information}

Precoding at transmitter based on channel state can achieve channel capacity and/or BER performance. Contrary to solely detection-based methods, CSI requires transmitter-side processing but not necessarily receiver-side one.

In order to take advantage of CSI at transmitter, several schemes can be applied to the signals to be transmitted. The simplest and most referenced one is bit-loading, based on waterfilling principles. In a bit-loading scheme, a constraint algorithm based on channel state and available transmit power is applied to decide how to modulate each sub-carrier, given a set of possible modulations. In this paper, a bit-loading algorithm is applied on top of OFDM.

In the presence of MAI, as in OFDM-CDM transmissions, when channel matrix can no longer be considered as diagonal, joint processing techniques can be applied in order to regain a diagonal channel matrix. In this paper, a technique based on the Singular Value Decomposition (SVD) of the equivalent channel matrix is applied to OFDM-CDM communications.

\subsection{Application of bit-loading to OFDM}

When transmitter has knowledge of the channel, it can use different modulations schemes for each sub-carriers and/or distribute available transmit power among sub-carriers.

In a previous work, a scheme of power allocation over OFDM based on BER minimisation was studied [4]. There, it was shown power allocation on its own cannot overcome the stringent requirements of the HF channel, as deep nulls will cause the loss of the data in the sub-carriers affected by them. Thus, in this paper a bit-loading algorithm is studied, also based on BER minimisation. Specifically, an algorithm developed by Fisher and Huber has been chosen [2].
The bit-loading algorithm selected allows applying a different modulation scheme to each sub-carrier. Thus, sub-carriers most affected by channel are assigned less dense constellations. As long as bit rate per OFDM symbol is a constant, it is even possible that no data is assigned to a given sub-carrier. Lastly, once a modulation scheme has been decided for all sub-carriers, available power is distributed amongst them with the added constraint that all sub-carriers yield the same error probability.

\subsection{Application of SVD to OFDM-CDM}

Channel matrix decomposition by means of SVD is a straightforward joint processing scheme that allows taking advantage of CSI at both transmitter and receiver [7]. This technique is widely known and applied in the context of Multiple Input Multiple Output (MIMO) transmissions. However, this paper focuses on Single Input Single Output (SISO) transmissions. Thus, the application of SVD to SISO conditions is based on the view of regarding the different spreaded symbols in an OFDM-CDM communication as a kind of multiple access. Associating the spreading matrix with the channel gives rise to a non-diagonal equivalent channel matrix, which in turn can be diagonalised.

In this context, the equivalent channel matrix is given by

$$
\mathbf{H}_{\mathrm{eq}}=\mathbf{H C} \text {, }
$$

where $\mathbf{H}$ and $\mathbf{C}$ are channel matrix and spreading matrix, respectively. Thus, $\mathbf{H}_{\mathrm{eq}}$ can be obtained as the product of three matrices as

$$
\mathbf{H}_{\mathrm{eq}}=\mathbf{U} \boldsymbol{\Sigma} \mathbf{V}^{\mathrm{H}}
$$

with $\Sigma$ diagonal of singular values and $\mathbf{U}$ and $\mathbf{V}$ unitary matrices. $\mathbf{V}^{\mathrm{H}}$ denotes the conjugate transpose of matrix $\mathbf{V}$. If vector to be transmitted is $\mathbf{s}$, transmitter processing is given by

$$
\mathbf{x}=\mathbf{V s},
$$

where $\mathbf{x}$ is the actual transmitted signal. If the received signal is

$$
\mathbf{y}=\mathbf{H}_{\mathrm{eq}} \mathbf{x}+\mathbf{n},
$$

with $\mathbf{n}$ white Gaussian noise vector, proper receiver processing in order to recover $\hat{\mathbf{s}}$ as estimation for transmitted $\mathbf{s}$ is given by

$$
\hat{\mathbf{s}}=\Sigma^{+} \mathbf{U}^{\mathrm{H}} \mathbf{y}
$$

where $\Sigma^{+}$denotes pseudoinverse of $\Sigma$. Substituting Equations (2) and (4) into Equation (5) yields

$$
\hat{\mathbf{s}}=\boldsymbol{\Sigma}^{+} \mathbf{U}^{\mathrm{H}} \mathbf{U} \boldsymbol{\Sigma} \mathbf{V}^{\mathrm{H}} \mathbf{V} \mathbf{s}+\Sigma^{+} \mathbf{U}^{\mathrm{H}} \mathbf{n}=\mathbf{s}+\mathbf{n}^{\prime} .
$$

It can be proven that n' gives rise to parallel AWGN uncorrelated channels [7].

\section{Non-ideal Channel State Information}

There are several sources of non-ideality in CSI that can be taken into consideration, such as error in channel estimations, quantisation and error in channel update from receiver to transmitter, and delay in application of channel knowledge at transmitter, amongst others. The net effect on communication is a loss in system performance. 
Of all the possible sources of non-ideality, this paper will pursue delay in application of channel estimation at transmitter-side. The decrease in performance is a function of both channel characteristics and delay length.

\subsection{Effect of delay bit-loading}

As channel applied by transmitter departs from the actual channel, performance decreases, as the modulation selected for each sub-carrier will no longer match the state of the channel. It is expected that as this mismatch between transmitter-applied and actual channel increases, performance tends to that of plain OFDM, as it basically corresponds with a situation of a randomly selection of modulation per subcarrier.

Moreover, as a transmitter-side scheme, receiver cannot compensate for the error committed by transmitter when selecting the modulation for each sub-carrier with a channel other than the actual one.

\subsection{Effect of delay on SVD}

When transmitter applies a channel different to the one currently present, a loss of orthogonality in SVD takes place. To overcome this loss of orthogonality, a compensation technique can be applied to eliminate the error caused by matrix mismatch between transmitter and receiver [5].

If actual channel in a given instant is $\mathbf{H}_{\mathrm{t}}$ and the delay in application of channel at transmitter is $\mathrm{T}$, at that instant transmitter applies to SVD processing channel $\mathbf{H}_{\mathrm{t}-\mathrm{T}}$. Thus, similarly to Equation (3), processing at transmitter is given by

$$
\mathbf{x}=\mathbf{V}_{\mathrm{t}-\mathrm{T}} \mathbf{S} \text {. }
$$

Thus, operating the same way that led to Equation (6), the received signal after processing at receiver by

is given by

$$
\hat{\mathbf{s}}=\Sigma_{\mathrm{t}}^{+} \mathbf{U}_{\mathrm{t}}^{\mathrm{H}} \mathbf{y}
$$

$$
\hat{\mathbf{s}}=\boldsymbol{\Sigma}_{\mathrm{t}}^{+} \mathbf{U}_{\mathrm{t}}^{\mathrm{H}} \mathbf{U}_{\mathrm{t}} \boldsymbol{\Sigma}_{\mathrm{t}} \mathbf{V}_{\mathrm{t}}^{\mathrm{H}} \mathbf{V}_{\mathrm{t}-\mathrm{T}} \mathbf{s}+\boldsymbol{\Sigma}_{\mathrm{t}}^{+} \mathbf{U}_{\mathrm{t}}^{\mathrm{H}} \mathbf{n}=\mathbf{V}_{\mathrm{t}}^{\mathrm{H}} \mathbf{V}_{\mathrm{t}-\mathrm{T}} \mathbf{s}^{+} \mathbf{n}^{\prime} .
$$

Thus, in order to compensate for the mismatch between matrices $\mathbf{V}_{\mathrm{t}}$, corresponding to the actual channel, and $\mathbf{V}_{\mathrm{t}-\mathrm{T}}$, corresponding to the channel applied by transmitter, as shown in Equation (9), receiver must invert both of them. Finally, it can be easily seen that proper receiver-side processing in order to achieve the same result than that of Equation (6), is then not given by Equation (8), but by

$$
\hat{\mathbf{s}}=\mathbf{V}_{\mathrm{t}-\mathrm{T}}{ }^{\mathrm{H}} \mathbf{V}_{\mathrm{t}} \boldsymbol{\Sigma}_{\mathrm{t}}^{+} \mathbf{U}_{\mathrm{t}}^{\mathrm{H}} \mathbf{y} \text {. }
$$

\section{Simulations}

To carry out the simulations, a 60 sub-carriers OFDM signal, transmitted at a rate of 1/30 seconds per OFDM symbol has been employed. In the case of OFDM-CDM transmissions, a load of approximately $2 / 3$ has been chosen, in order to achieve spreading gain. Thus, 41 symbols are spreaded over the 60 available sub-carriers. In order to keep the same overall bit rate for both transmission schemes, when plain OFDM is simulated, only 41 of the 60 sub-carriers are used.
In the case of transmissions with constant bit rate per subcarrier, a QPSK modulation has been selected, corresponding to a bit rate of 82 bits per OFDM symbol. This rate corresponds to a net rate of 2460 bits per second, as used in the interactive digital voice modem developed by the researchers' groups. By using this rate, a vocoder link can be established to carry out the communication, such as the Mixed Excitation Linear Prediction (MELP) rated to 2400 bits per seconds.

In the simulations where a bit-loading scheme is applied, a variable $n \mathrm{PSK}$ modulation is to be selected by the algorithm, ranging from BPSK to 16-QPSK, or equivalently a load of 1 to 4 bits per sub-carrier, or it can even leave a sub-carrier unmodulated. Furthermore, the bit-loading algorithm is constrained to preserve the overall rate than that used by constant bit rate schemes, precisely 82 bits per OFDM symbol.

In the case of OFDM transmissions, a Minimum Mean Square Error (MMSE) detector has been used at receiver, while for OFDM-CDM transmissions a MMSE plus a MUD detector has been used.

The source of non-ideality studied in this paper is the delay $\Delta$ in retransmission of the actual channel state from receiver to transmitter. Transmitter receives an updated channel from receiver, and applies that same channel for bit-loading or SVD until a new channel is received, regardless of the current state of the actual channel.

\subsection{HF Channel Model}

Typical values for frequency coherence in the HF channel are about hundreds of $\mathrm{Hz}$ or less. Doppler spread usually varies between 0.1 and $2 \mathrm{~Hz}$, while time spread is usually found to be between 1 and $4 \mathrm{~ms}$, thus making efficiencies over 0.5 $\mathrm{bit} / \mathrm{Hz}$ difficult to achieve. It is not uncommon to find several deep nulls spread over the usually narrow transmission band, and to move arbitrarily over it. Thus, it is rather common to have available bandwidths smaller than $3 \mathrm{kHz}$.

For the present simulations, ITU-R recommendations for HF simulations have been selected [3]. Specifically, a moderate channel has been used, with a $0.5 \mathrm{~Hz}$ doppler spread and $1 \mathrm{~ms}$ time spread, with a noise bandwidth of $4.8 \mathrm{kHz}$.

\subsection{Results}

Due mainly to the doubly dispersive nature of the HF channel, in the range of SNR values considered for these simulations, OFDM and OFDM-CDM perform almost identically, as shown in Figure 1. However, OFDM reaches a lower bound on BER achieved, as data in sub-carriers affected by deep nulls cannot be recovered by the receiver and it is irremediably lost. 


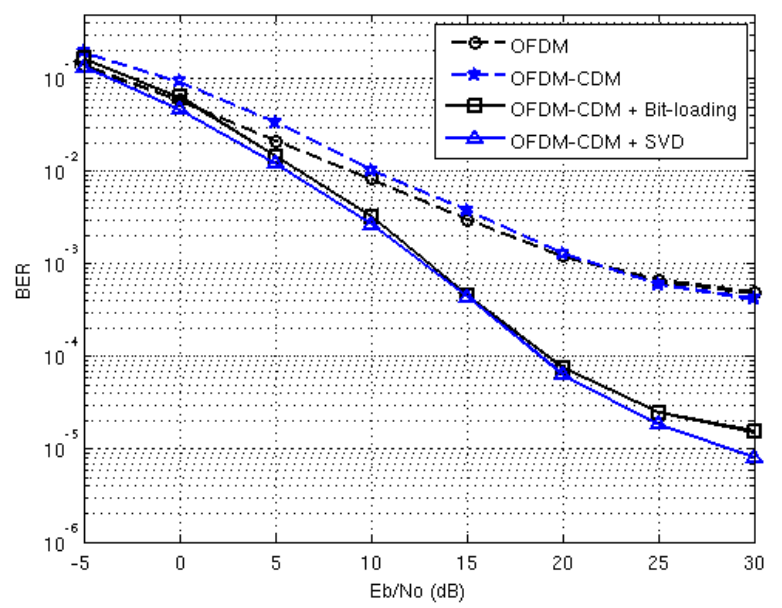

Figure 1: Performance comparison for OFDM and OFDMCDM under ideal CSI.

When the bit-loading algorithm is applied, performance of OFDM increases dramatically, as the loading algorithm is able to avoid sub-carriers subject to deep nulls, increasing the load on those less affected by the channel, as depicted in Figure 1. In the case of OFDM-CDM with SVD decomposition applied, also shown in Figure 1, performance also increases as MAI can be overcome thanks to separation of the spreaded symbols on independent channels. Moreover, under these ideal CSI conditions, OFDM and OFDM-CDM achieve virtually the same performance, as once deep nulls and MAI effects have been overcome, both share a common underlying nature.

Figure 2 shows the effect of delay on CSI application at transmitter for OFDM communications. $\Delta$ corresponds to the number of OFDM symbols transmitted without a channel update from the receiver. Three values have been depicted: 5 , 10 and 15; with the channel parameters selected for the simulations, those values correspond to a $33.3 \%, 66.7 \%$ and $100 \%$ of the channel coherence time, respectively. As delay increases, performance decreases, quickly approaching that of OFDM without bit-loading applied, as modulation per subcarrier no longer matches the actual state of the channel. Once a sufficiently long delay is reached, performance of bitloading is similar to that of a random selection of the modulation scheme.

Figure 3 shows how delay on CSI application affects OFDMCDM communications. $\Delta$ has the same aforementioned meaning. Thus, delays correspond with a $33.3 \%, 66.7 \%$ and $100 \%$ of the channel coherence time. Performance drops abruptly, even for the lowest delay considered, as the mismatch between channel applied by transmitter and actual channel, greatly affects SVD diagonalisation. Thus, values outside of the main diagonal of channel matrix arise, causing interference amongst the different spreaded symbols.

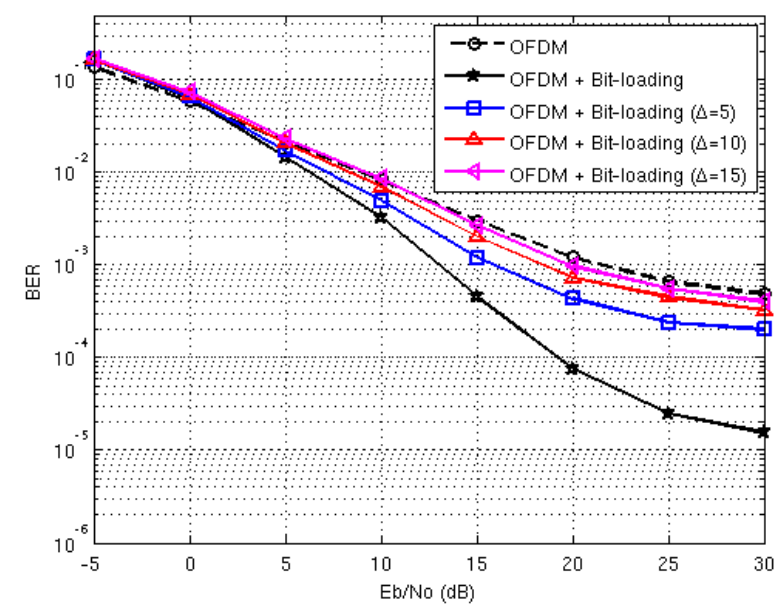

Figure 2: Performance for OFDM with delay in application of channel knowledge at transmitter.

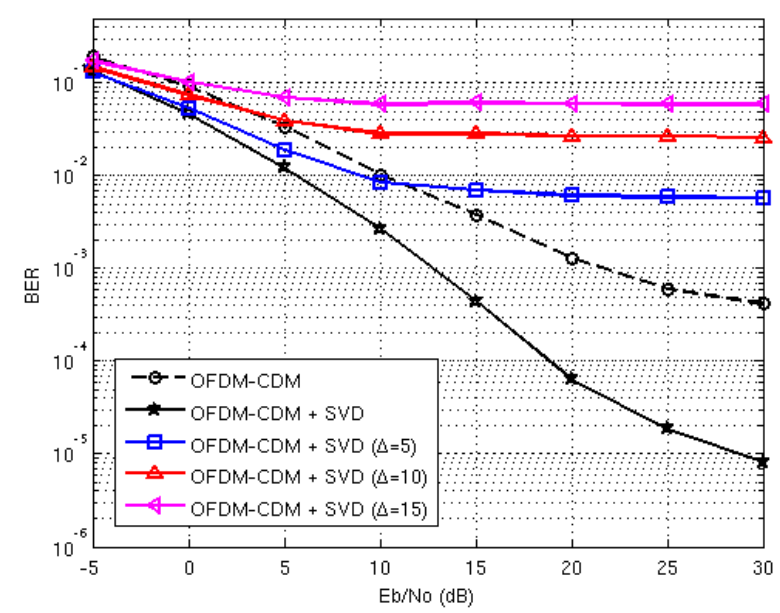

Figure 3: Performance for OFDM-CDM with delay in application of channel knowledge at transmitter.

However, if the correction technique introduced earlier is applied, as shown in Figure 4 where this correction technique is labelled as SVD2, receiver can compensate for the mismatch in the matrices applied by transmitter with those of the SVD decomposition of the actual channel. As figure shows, performance practically matches that of ideal CSI, for all the range of delays considered.

These simulations highlight the common underlying nature of OFDM and OFDM-CDM. In the case of ideal CSI, once deep nulls and MAI have been avoided, performance of both techniques is almost equivalent; however, OFDM-CDM has the added complexity of joint processing requirements instead of only transmitter-side ones, as in the case of OFDM. When delay in CSI application is taken into account, performance of OFDM tends gradually to that of OFDM without bit-loading, while OFDM-CDM performance drops abruptly. However, receiver can easily compensate for the mismatch in the matrices causing the loss of performance, bringing it up to par with that of ideal CSI. 


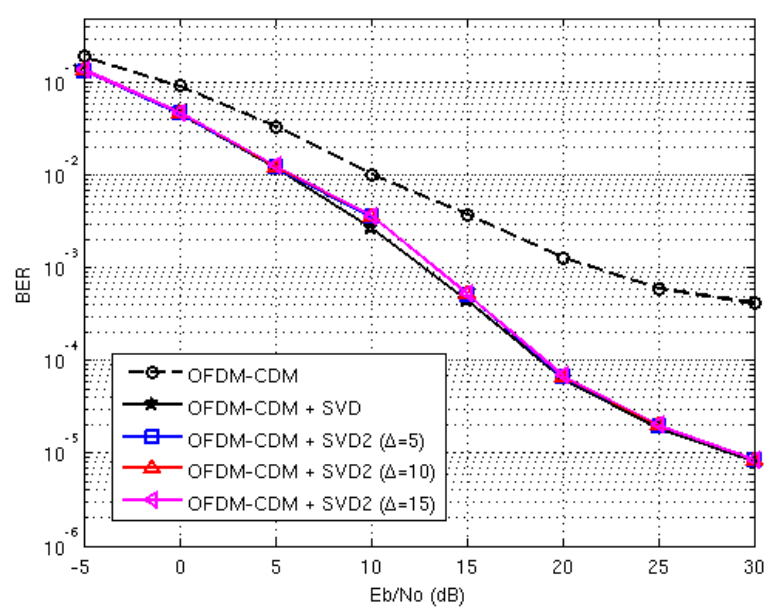

Figure 4: Performance for OFDM-CDM with delay in application of channel knowledge at transmitter when correction technique applied by receiver.

\section{Conclusions}

In this paper, usage of channel state knowledge at transmitter to sensitive-delay applications on the HF band has been shown. OFDM and OFDM-CDM have been chosen as communications schemes, with two CSI techniques on top: bit-loading over OFDM and SVD over OFDM-CDM. Moreover, impact of CSI latency on performance has been studied.

In case of ideal CSI, it has been shown that if a bit-loading scheme is applied to OFDM, and channel matrix SVD decomposition to OFDM-CDM, performance increases significantly. Furthermore, due to the common underlying nature of both OFDM and OFDM-CDM transmission schemes, they behave almost identically once the undesired effects of deep nulls and MAI are avoided. In this case, OFDM plus bit-loading can be a better alternative to OFDMCDM plus SVD, as it is a more simple technique that does not incur in any MAI, and also because only transmitter-side precoding is needed.

If non-ideal CSI is taken into consideration, by means of delay in application of channel knowledge by transmitter, performance decreases differently for OFDM than for OFDM-CDM. OFDM performance decreases gradually as delay increases, with a worst case scenario equal to performance of plain OFDM without channel state knowledge. On the other hand, OFDM-CDM performance drops abruptly, as SVD decomposition is highly sensitive to mismatch in the involved matrices. However, a simple correction technique can be applied at receiver, restoring performance close to that of ideal CSI. Under these more real conditions, OFDM-CDM can a better alternative to OFDM, as performance can be made close to that of ideal CSI.

\section{Acknowledgements}

The authors wish to thank Aeropuertos Nacionales y Navegación Aérea (AENA) for their support and also the Spanish Educational and Science Ministry under grants TEC2007-67520-C02-01/02/TCM and TEC2008-06874-C0303.

\section{References}

[1] K. Fazel and S. Kaiser, Multi-Carrier and Spread Spectrum Systems. New York: John Wiley and Sons, (2003).

[2] R. Fisher and J. Huber, "A new loading algorithm for discrete multitone transmission". Proceeding - Global Telecommunications Conference (GLOBECOM), 1, pp. 724-728, (1996).

[3] ITU-R F.1487. "Testing of HF modems with bandwidths of up to about $12 \mathrm{kHz}$ using ionospheric channel simulators". ITU-R Technical Report, (2000).

[4] J. López-Pérez, S. Zazo-Bello, I. Pérez-Álvarez, I. Raos and E. Mendieta-Otero, "Multi-carrier techniques performance on ionospheric channel for delay-sensitive applications". Proceedings - IEEE Military Communications Conference (MILCOM), 1, pp. 1-5, (2007).

[5] M. Tesanovic, D. Bull, A. Doufexi, V. Sgardoni and A. Nix. "Impact of CSI latency on video quality in MIMO systems employing singular value decomposition". IEEE Electronic Letters, 43 (18), pp. 972-973, (2007).

[6] S. Verdú, Multiuser Detection. New York: Cambridge University Press (1998).

[7] C. Windpassinger, "Detection and precoding for multiple input multiple output channels". Ph.D. Dissertation. Germany: Universität Erlangen-Nürnberg, (2004).

[8] S. Zazo-Bello and E. Esteban. "Comparison of single carrier and multicarrier techniques for the HFDL system". Sixth Meeting of the Aeronautical Mobile Communication Panel (AMCP), Montreal, (1999).

[9] S. Zazo-Bello, I. Raos, I. Pérez-Álvarez, H. SantanaSosa, E. Mendieta-Otero and J. López-Pérez. "MIMO transmission in the frequency domain over the HF channel". IET Conference Publications, 2006 (CP517), pp. 202-206, (2006). 